\title{
Isolation of phenolic constituents from Rhododendron yunnanense flowers as a potent cyclooxygenase- 2 and vascular endothelial growth factor receptor-2 inhibitor: Phytochemical and molecular simulation studies
}

\author{
Mohamed Salaheldin Ahmed Abdelkader ${ }^{1 *}$, Reda Ahmed Abdelhamid², Mohamed Ezzat Abouelela², Mostafa Ezzat Rateb ${ }^{3}$, \\ Marwa Hassan Ahmed ${ }^{4}$ \\ ${ }^{1}$ Department of Pharmacognosy, Faculty of Pharmacy, Sohag University, Sohag, Egypt. \\ ${ }^{2}$ Department of Pharmacognosy, Faculty of Pharmacy, Al-Azhar University, Assiut, Egypt. \\ ${ }^{3}$ Microbial Natural Product Laboratory, School of Computing, Engineering \& Physical Sciences, University of the West of Scotland, Paisley, UK. \\ ${ }^{4}$ Department of Pharmacognosy, Faculty of Pharmacy, Beni-Suef University, Beni-Suef, Egypt.
}

\author{
ARTICLE INFO \\ Received on: 04/05/2021 \\ Accepted on: 25/07/2021 \\ Available Online: 03/11/2021 \\ Key words: \\ Rhododendron yunnanense, \\ phenolics, quercitrin, \\ molecular docking, VEGF-2, \\ COX-2.
}

\begin{abstract}
The genus Rhododendron is a rich source of phenolic compounds that possess a wide range of biological activities. Phytochemical investigation of the methanolic extract of the flowers of Rhododendron yunnanense Franch. led to the isolation and characterization of 13 phenolic compounds isolated for the first time from this plant species. These compounds were identified as quercetin (1), quercitrin (2), avicularin (3), taxifolin-3-O- $\alpha$-L-arabinoside (4), azalein (5), kaempferol-3-O-rhamnoside (6), kaempferol-4'-methoxy-3-O-rhamnoside (7), kaempferol-3- $\beta$-Dglucopyranoside (8), catechin (9), epicatechin (10), catechin-3-O-gallate (11), 5-O-Z-p-coumaroylquinic acid methyl ester (12), and 5-O-caffoeylquinic acid methyl ester (13). The structures of compounds 1-13 were determined by $1 \mathrm{D}$ and 2D nuclear magnetic resonance and comparison with reported spectral data. A molecular simulation study was carried out on the binding mode of the isolated compounds as anti-inflammatory agents through cyclooxygenase 2 $(\mathrm{COX}-2)$ inhibition and as mediators of tumor angiogenesis through vascular endothelial growth factor inhibition. The docking results of the isolated compounds revealed promising binding affinities to the examined enzymes. Compound 2 showed predominant affinity for the two examined receptors [COX-2 $(-19.4542 \mathrm{kcal} / \mathrm{mol})$ and vascular endothelial growth factor receptor $2(-17.6036 \mathrm{kcal} / \mathrm{mol})]$. The isolated compounds offered significantly active phytoconstituents for drug discovery and development.
\end{abstract}

\section{INTRODUCTION}

Natural polyphenols are widely distributed phytochemicals in the plant kingdom that are considered a significant source for drug discovery and development (Asuzu et al., 2019). Recently, natural bioactive phenolics have gotten more attention as therapeutic agents due to their diverse bioactive

\footnotetext{
*Corresponding Author

Mohamed Salaheldin Ahmed Abdelkader, Department of Pharmacognosy, Faculty of Pharmacy, Sohag University, Sohag, Egypt. E-mail: m.salaheldin@pharm.sohag.edu.eg
}

functions, which potentially have beneficial implications in the underlying biological process in several diseases' regulation (Abhinand et al., 2020; Sayed et al., 2020). The treatment of cancer and chemopreventive and chemotherapeutic effects of polyphenolics, especially flavonoids, are intensively studied due to their low toxicity and antioxidant effects contributing to preventing and managing oxidative stress implicated mainly in cancer development (Asuzu et al., 2019).

The molecular targets for antineoplastic mechanisms include many pathways such as cell cycle arrest, apoptosis, necrosis, and angiogenesis inhibition (Jafari et al., 2014; Ko and Auyeung, 2013). Angiogenesis plays an essential role in 
increasing vasculature and blood supply needed for the growth, progression, and metastasis of tumors. Recent studies have shown that many isolated natural compounds exhibited antiangiogenic activity as a potential target for cancer treatments especially for solid tumors (Al Abd et al., 2017). It is well established that chronic inflammation is a risk factor for cancer development which is indicated by overexpression of inflammatory mediators such as cytokines, NF-kB, cyclooxygenase (COX), and vascular endothelial growth factor (VEGF) associated with various cancers development such as pancreatic, prostate, cervical, breast, lung, and colon cancers (Wong, 2019). Despite the fact that angiogenesis is regulated by multiple complex proangiogenic factors, the VEGF is a vital mediator of tumor angiogenesis in which its expression increases associated with tumor prognosis (Abhinand et al., 2020). Moreover, the expression of cyclooxygenase 2 (COX-2) by the tumor cells, particularly endothelial cells, was found to stimulate angiogenesis through the formation of prostaglandin E2 and interleukin-6 induction (Fosslien, 2000; Leahy et al., 2002). Recent studies suggest that COX-2 inhibitors could lead to a reduction of tumorigenesis by suppression of angiogenesis through the downregulation of VEGF production and other proangiogenic factors produced by tumor cells (Liu et al., 2000; Toomey et al., 2009).

The genus Rhododendron belongs to the family Ericaceae which comprises more than 1,000 species, which are widely distributed all over the world (Popescu and Kopp, 2013). Rhododendron plants have been used in ancient traditional Chinese, Ayurvedic, European, and North American folk medicine (Popescu and Kopp, 2013). It possesses massive biological effects as anti-inflammatory, analgesic (Verma et al., 2010), antibacterial (Chhetri et al., 2008; Silici et al., 2010), antifungal (Jin et al., 1999), antiprotozoal (Tasdemir et al., 2005), antiviral (Zheng, 1989), antioxidant (Silici et al., 2010; Takahashi et al., 2001), and cytotoxic activities (Rateb et al., 2014). Furthermore, they have inhibitor activities on tyrosinase enzyme (Ahmad et al., 2004; Ullah et al., 2007) and acetylcholine esterase enzyme (Orhan et al., 2004). These biological activities have been owed mainly to the phenolic and flavonoid contents of different Rhododendron spp. (Popescu and Kopp, 2013; Verma et al., 2010). Furthermore, the majority of isolated compounds have been previously evaluated for COX-1, COX-2, and vascular endothelial growth factor receptor (VEGFR) inhibitory activity (Adhikari et al., 2006; Bahamonde et al., 2013; Löhr et al., 2015; Noreen et al., 1998, Popescu and Kopp, 2013; Riaz et al., 2018; Roleira et al., 2015; Sung et al., 2012; Valero et al., 2020; Wang et al., 2019; Zhang et al., 2020). Herein, our study is focused on the isolation and identification of phenolic and flavonoid constituents of Rhododendron yunnanense Franch. flowers and investigation of the anti-inflammatory (COX2) and antiangiogenic effects (VEGF) through in silico molecular docking to identify the affinity and interactions mode of isolated compounds toward the targeted receptors.

\section{MATERIAL AND METHODS}

\section{General experimental procedures}

${ }^{1} \mathrm{H}$ and ${ }^{13} \mathrm{C}$ nuclear magnetic resonance (NMR) spectra were recorded at $25^{\circ} \mathrm{C}$ with a Varian Inova $600 \mathrm{MHz}$ NMR spectrometer. High-pressure liquid chromatography (HPLC) was carried out on Agilent 1260 Infinity preparative HPLC system with an Agilent Eclipse XDB-C18 column ( 5 m, $10 \times 250$ mm, Agilent Technologies, USA) monitored using an Agilent photodiode array detector. Detection was carried out at 220,254, 280, 350, and 400 $\mathrm{nm}$. All chemical reagents were purchased from Sigma-Aldrich and used without further purification. Medium-pressure liquid chromatography (MPLC) separations were carried out on Biotage system using Biotage reversed-phase and normal-phase silica prepacked columns. Detection was carried out at 220 and $280 \mathrm{~nm}$. TLC was carried out on precoated TLC plates with silica gel 60 F254 (layer thickness $0.2 \mathrm{~mm}$, Merck, Darmstadt, Germany).

\section{Plant material}

The flower of the plant was collected in May 2015 from the University of Aberdeen Botanical Garden, Aberdeen, UK. The plant was labeled by its name by the authority of the garden. The plant flowers were collected and dried under shade and finely powdered and the powder was used for the extraction procedure.

\section{Extraction and isolation}

The air-dried powdered $R$. yunnanense flower $(0.3 \mathrm{~kg})$ was extracted with methanol $(\mathrm{MeOH})(4 \times 11)$. The methanolic extract was evaporated and concentrated under reduced pressure to afford a dark yellowish-green residue $(25 \mathrm{~g})$. The latter was suspended in distilled water $(500 \mathrm{ml})$ and then successively partitioned among $n$-hexane $(500 \mathrm{ml} \times 4), \mathrm{CHCl}_{3}(500 \mathrm{ml} \times 4)$, and EtOAc $(500 \mathrm{ml} \times 4)$, and each fraction was concentrated under reduced pressure to give $n$-hexane $(2.8 \mathrm{~g}), \mathrm{CHCl}_{3}(3.8$ g), EtOAc (6.9 g), and aqueous ( $7.0 \mathrm{~g})$ extracts, respectively. Approximately $5 \mathrm{~g}$ of the EtOAc fraction was subjected to vacuum liquid chromatography using $\mathrm{CHCl}_{3} / \mathrm{MeOH}$ gradients to obtain five subfractions: fraction $\mathrm{A}(0.9 \mathrm{~g})$, fraction $\mathrm{B}(0.7 \mathrm{~g})$, fraction C $(0.9 \mathrm{~g})$, fraction D $(1.5 \mathrm{~g})$, and fraction E $(0.8 \mathrm{~g})$. Fraction A was subjected to MPLC in the Biotage system using prepacked RP-18 column chromatography with $\mathrm{MeOH} / \mathrm{H}_{2} \mathrm{O}$ gradients, followed by semipreparative RP-HPLC $\left(\mathrm{MeOH} / \mathrm{H}_{2} \mathrm{O}\right)$ gradients to afford compounds 9 (10 mg), $10(7 \mathrm{mg})$, and $11(6 \mathrm{mg})$. Similarly, fraction B afforded compound $13(10 \mathrm{mg})$ and fraction C afforded compounds $3(8 \mathrm{mg})$ and $12(12 \mathrm{mg})$. Fraction D was subjected to MPLC using the Biotage system on prepacked RP-18 column chromatography $\left(\mathrm{MeOH} / \mathrm{H}_{2} \mathrm{O}\right)$, followed by semipreparative RPHPLC $\left(\mathrm{MeOH} / \mathrm{H}_{2} \mathrm{O}\right)$ gradients to afford compounds $2(5 \mathrm{mg}), 3$ (10 mg), 4, (8 mg), 5 (6 mg), 6 (12 mg), 7 (10 mg), and 8 (15 mg). Finally, fraction E was similarly treated as fraction D to afford compound $1(10 \mathrm{mg})$.

Quercetin (1): yellow crystal, ${ }^{1} \mathrm{H}$ NMR (DMSO- $d_{6}, 600$ MHz): $\delta 6.18,(1 \mathrm{H}, \mathrm{d}, J=1.98 \mathrm{~Hz}, \mathrm{H}-6), 6.40(1 \mathrm{H}, \mathrm{d}, J=2.04 \mathrm{~Hz}$, $\mathrm{H}-8), 7.67\left(1 \mathrm{H}, \mathrm{d}, J=1.98 \mathrm{~Hz}, \mathrm{H}-2^{\prime}\right), 6.88(1 \mathrm{H}, \mathrm{d}, J=8.40 \mathrm{~Hz}$, H-5'), 7.54 (1H, dd, $\left.J=2.06,8.34 \mathrm{~Hz}, \mathrm{H}-6^{\prime}\right) .{ }^{13} \mathrm{C}$ NMR (DMSO- $d_{6}$, $150 \mathrm{MHz}): \delta 157.3$ (C-2), 134.2 (C-3), 177.7 (C-4), 161.3 (C-5), 98.7 (C-6), 164.3 (C-7), 93.6 (C-8), 156.5 (C-9), 104.1 (C-10), $120.8\left(\mathrm{C}-1^{\prime}\right), 115.7\left(\mathrm{C}-2^{\prime}\right), 145.2\left(\mathrm{C}-3^{\prime}\right), 148.5\left(\mathrm{C}-4^{\prime}\right), 115.5\left(\mathrm{C}-5^{\prime}\right)$, $121.1\left(\mathrm{C}-6^{\prime}\right)$.

Quercetin 3- $\alpha$-L-rhamnoside "Quercitrin" (2): yellow powder, ${ }^{1} \mathrm{H}$ NMR (DMSO- $\left.d_{\sigma}, 600 \mathrm{MHz}\right): \delta 6.20,(1 \mathrm{H}, \mathrm{d}, J=1.98$ $\mathrm{Hz}, \mathrm{H}-6), 6.39(1 \mathrm{H}, \mathrm{d}, J=2.04 \mathrm{~Hz}, \mathrm{H}-8), 7.30(1 \mathrm{H}, \mathrm{d}, J=1.92 \mathrm{~Hz}$, H-2'), $6.87\left(1 \mathrm{H}, \mathrm{d}, J=8.40 \mathrm{~Hz}, \mathrm{H}-5^{\prime}\right), 7.25(1 \mathrm{H}, \mathrm{dd}, J=2.2,8.34$ Hz, H-6') 5.26 (1H, br.s, H-1"), 3.97 (1H, br.s, H-2"), 3.51 (1H, dd, 
$\left.J=6.06,3.12 \mathrm{~Hz}, \mathrm{H}-3^{\prime \prime}\right), 3.14\left(1 \mathrm{H}, \mathrm{d}, J=9.42 \mathrm{~Hz}, \mathrm{H}-4^{\prime \prime}\right), 3.22(1 \mathrm{H}$, m, H-5"), $0.82\left(3 \mathrm{H}, \mathrm{d}, J=6.12 \mathrm{~Hz}, \mathrm{H}-6{ }^{\prime \prime}\right) .{ }^{13} \mathrm{C}$ NMR (DMSO- $d_{6}$, $150 \mathrm{MHz}): \delta 157.3$ (C-2), 134.2 (C-3), 177.7 (C-4), 161.3 (C-5), 98.7 (C-6), 164.3 (C-7), 93.6 (C-8), 156.5 (C-9), 104.1 (C-10), $120.8\left(\mathrm{C}-1^{\prime}\right), 115.7\left(\mathrm{C}-5^{\prime}\right), 145.2\left(\mathrm{C}-3^{\prime}\right), 148.5$ (C-4'), $115.5\left(\mathrm{C}-2^{\prime}\right)$, $121.1\left(\mathrm{C}-6^{\prime}\right), 102.3\left(\mathrm{C}-1^{\prime \prime}\right), 70.4\left(\mathrm{C}-2^{\prime \prime}\right), 70.6\left(\mathrm{C}-3^{\prime \prime}\right), 71.2\left(\mathrm{C}-4^{\prime \prime}\right)$, $70.1\left(\mathrm{C}-5^{\prime \prime}\right), 17.5\left(\mathrm{C}-6^{\prime \prime}\right)$

Quercetin-3- $O-\alpha$-L- arabinofuranoside "Avicularin" (3): yellow powder, 1H NMR (DMSO- $d, 600 \mathrm{MHz}): \delta 6.20,(1 \mathrm{H}, \mathrm{d}$, $J=2.0 \mathrm{~Hz}, \mathrm{H}-6), 6.40(1 \mathrm{H}, \mathrm{d}, J=2.0 \mathrm{~Hz}, \mathrm{H}-8), 7.55$ (1H, dd, $J$ $\left.=2.20,8.40 \mathrm{~Hz}, \mathrm{H}-6^{\prime}\right), 7.48\left(1 \mathrm{H}, \mathrm{d}, J=2.2 \mathrm{~Hz}, \mathrm{H}-2^{\prime}\right), 6.85(1 \mathrm{H}$, $\left.\mathrm{d}, J=8.41 \mathrm{~Hz}, \mathrm{H}-5^{\prime}\right), 5.59\left(1 \mathrm{H}, \mathrm{d}, J=1.2 \mathrm{~Hz}, \mathrm{H}-1^{\prime \prime}\right), 3.72(1 \mathrm{H}, \mathrm{m}$, H-2"), $3.56\left(1 \mathrm{H}, \mathrm{m}, \mathrm{H}-3^{\prime \prime}\right), 4.15$ (1H, dd, $\left.J=1.38,4.68 \mathrm{~Hz}, \mathrm{H}-4^{\prime \prime}\right)$, $3.30(2 \mathrm{H}, \mathrm{m}, \mathrm{H}-5 ") .{ }^{13} \mathrm{C}$ NMR (DMSO- $\left.d_{6}, 150 \mathrm{MHz}\right): \delta 156.8(\mathrm{C}-$ 2), 133.8 (C-3), 177.7 (C-4), 161.2 (C-5), 98.9 (C-6), 164.2 (C-7), 93.2 (C-8), 156.2 (C-9), 103.8 (C-10), 120.8 (C-1'), 115.2 (C-2'), $144.9\left(\mathrm{C}-3^{\prime}\right), 148.3\left(\mathrm{C}-4^{\prime}\right), 115.2\left(\mathrm{C}-5^{\prime}\right), 121.4\left(\mathrm{C}-6^{\prime}\right), 107.5(\mathrm{C}-$ $\left.1^{\prime \prime}\right), 76.5$ (C-3"), 85.5 (C-4"), 81.8 (C-2"), 60.8 (C-5").

Taxifolin-3- $O-\beta$-L- arabinopyranoside (4): yellow powder, ${ }^{1} \mathrm{H}$ NMR (DMSO- $\left.d_{6}, 600 \mathrm{MHz}\right): \delta 5.44$, $(1 \mathrm{H}, \mathrm{d}, J=6.60$ Hz, H-2), 4.59 (1H, d, $J=6.60 \mathrm{~Hz}, \mathrm{H}-3), 5.85$, (1H, br.s, H-6), 5.88 (1H, br.s, H-8), $6.79\left(1 \mathrm{H}, \mathrm{d}, J=2.00 \mathrm{~Hz}, \mathrm{H}-2^{\prime}\right), 6.70(1 \mathrm{H}, \mathrm{d}, J$ $\left.=8.10 \mathrm{~Hz}, \mathrm{H}-5^{\prime}\right), 6.65\left(1 \mathrm{H}, \mathrm{dd}, J=1.62,8.28 \mathrm{~Hz}, \mathrm{H}-6^{\prime}\right) 4.09(1 \mathrm{H}$, d, $\left.J=6.90 \mathrm{~Hz}, \mathrm{H}-1^{\prime \prime}\right), 3.03$ (1H, m, H-2"), 3.27 (1H, m, H-3"), 2.95 (1H, m, H-4"), 3.72 (1H, m, H-5"a), 2.95 (1H, m, H-5"b). ${ }^{13} \mathrm{C}$ NMR (DMSO- $d, 150 \mathrm{MHz}$ ): $\delta 80.7$ (C-2), 76.1 (C-3), 192.1 (C-4), 163.4 (C-5), 95.6 (C-6), 167.6 (C-7), 95.2 (C-8), 161.3 (C9), 100.8 (C-10), 126.5 (C-1'), 114.4 (C-2'), 145.2 (C-3'), 145.6 (C-4'), $115.4\left(\mathrm{C}-5^{\prime}\right), 118.4\left(\mathrm{C}-6^{\prime}\right), 102.1\left(\mathrm{C}-1^{\prime \prime}\right), 72.7$ (C-2"), 69.2 $\left(\mathrm{C}-3^{\prime \prime}\right), 75.5\left(\mathrm{C}-4^{\prime \prime}\right), 65.3\left(\mathrm{C}-5^{\prime \prime}\right)$.

Azaleatin 3-O- $\alpha$-L-rhamnoside "Azalein" (5): pale yellow needles, ${ }^{1} \mathrm{H}$ NMR (DMSO- $\left.d_{6}, 600 \mathrm{MHz}\right): \delta 6.36(1 \mathrm{H}, \mathrm{d}$, $J=2.00 \mathrm{~Hz}, \mathrm{H}-6), 6.41(1 \mathrm{H}, \mathrm{d}, J=2.00 \mathrm{~Hz}, \mathrm{H}-8), 7.24$ (1H, d, $J=$ $\left.2.10 \mathrm{~Hz}, \mathrm{H}-2^{\prime}\right), 6.83\left(1 \mathrm{H}, \mathrm{d}, J=8.34 \mathrm{~Hz}, \mathrm{H}-5^{\prime}\right), 7.19$ (1H, dd, $J=$ $\left.2.00,8.28 \mathrm{~Hz}, \mathrm{H}-6^{\prime}\right) 5.20\left(1 \mathrm{H}, \mathrm{d}, J=1.32 \mathrm{~Hz}, \mathrm{H}-1^{\prime \prime}\right), 3.11(1 \mathrm{H}, \mathrm{m}$, H-2"), 3.49 (1H, br.s, H-3"), 3.12 (1H, m, H-4"), 4.00 (1H, br.s, H-5"), $0.78\left(3 \mathrm{H}, \mathrm{d}, J=5.40 \mathrm{~Hz}, \mathrm{H}-6^{\prime \prime}\right), 3.80(3 \mathrm{H}, \mathrm{s}, \mathrm{OCH} 3) .{ }^{13} \mathrm{C}$ NMR (DMSO- $\left.d_{6}, 150 \mathrm{MHz}\right): \delta 158.1$ (C-2), 136.3 (C-3), 172.0 (C-4), 160.8 (C-5), 94.7 (C-6), 162.5 (C-7), 92.2 (C-8), 158.1 (C9), 107.3 (C-10), $121.2\left(\mathrm{C}-1^{\prime}\right), 115.5\left(\mathrm{C}-2^{\prime}\right), 147.6\left(\mathrm{C}-3^{\prime}\right), 145.1$ (C-4'), 115.3 (C-5'), 120.7 (C-6'), $101.1\left(\mathrm{C}-1^{\prime \prime}\right), 70.3$ (C-2"), 70.4 (C-3"), 71.3 (C-4"), 70.1 (C-5"), 17.5 (C-6"), 55.9 (-OCH3).

Kaempferol-3-O- $\alpha$-L-rhamnoside (6): yellow powder, ${ }^{1} \mathrm{H}$ NMR (DMSO- $\left.d 6,600 \mathrm{MHz}\right): \delta 6.21(1 \mathrm{H}, \mathrm{d}, J=1.96 \mathrm{~Hz}, \mathrm{H}-6)$, $6.41(1 \mathrm{H}, \mathrm{d}, J=1.96 \mathrm{~Hz}, \mathrm{H}-8), 7.75\left(2 \mathrm{H}, \mathrm{d}, J=8.83 \mathrm{~Hz}, \mathrm{H}-2^{\prime}, 6^{\prime}\right)$, $6.91\left(2 \mathrm{H}, \mathrm{d}, J=8.83 \mathrm{~Hz}, \mathrm{H}-3^{\prime}, 5^{\prime}\right), 5.29$ (1H, br.s, H-1"), $3.08(1 \mathrm{H}$, m, H-2"), 3.97 (1H, m, H-3"), 3.47 (1H, m, H-4"), $3.12(1 \mathrm{H}, \mathrm{m}$, H-5"), 0.79 (3H, d, $\left.J=6.3 \mathrm{~Hz}, \mathrm{H}-66^{\prime \prime}\right) .{ }^{13} \mathrm{C}$ NMR (DMSO- $d 6,150$ MHz): $\delta 156.5$ (C-2), 134.2 (C-3), 177.7 (C-4), 157.2 (C-5), 98.7 (C-6), 164.2 (C-7), 93.7 (C-8), 161.3 (C-9), 104.1 (C-10), 120.5 $\left(\mathrm{C}-1^{\prime}\right), 130.6\left(\mathrm{C}-2^{\prime}, 6^{\prime}\right), 115.4\left(\mathrm{C}-3^{\prime}, 5^{\prime}\right), 159.9\left(\mathrm{C}-4^{\prime}\right), 101.8\left(\mathrm{C}-1^{\prime \prime}\right)$, 71.1 (C-2"), 70.0 (C-3"), 70.3 (C-4"), 70.6 (C-5"), 17.5 (C-6").

Kaempferol-4'-methoxy-3-O- $\alpha$-L-rhamnoside (7) yellow amorphous powder, ${ }^{1} \mathrm{H}$ NMR (DMSO- $\left.d 6,600 \mathrm{MHz}\right): \delta$ $6.21(1 \mathrm{H}, \mathrm{d}, J=1.98 \mathrm{~Hz}, \mathrm{H}-6), 6.41(1 \mathrm{H}, \mathrm{d}, J=1.98 \mathrm{~Hz}, \mathrm{H}-8), 7.69$ $\left(2 \mathrm{H}, \mathrm{d}, J=8.82 \mathrm{~Hz}, \mathrm{H}-2^{\prime}, 6^{\prime}\right), 6.88\left(2 \mathrm{H}, \mathrm{d}, J=8.83 \mathrm{~Hz}, \mathrm{H}-3^{\prime}, 5^{\prime}\right)$, $5.22\left(1 \mathrm{H}, \mathrm{s}, \mathrm{H}-1^{\prime \prime}\right), 3.01$ (1H, m, H-2"), 4.01 (1H, m, H-3"), 3.44
(1H, m, H-4"), 3.12 (1H, m, H-5"), 0.75 (3H, d, $J=3.54$ Hz, H-6"), $3.79(3 \mathrm{H}, \mathrm{s},-\mathrm{OCH} 3)$.

${ }^{13} \mathrm{C}$ NMR (DMSO- $\left.d, 150 \mathrm{MHz}\right): \delta 156.5(\mathrm{C}-2), 134.2$ (C-3), 177.7 (C-4), 157.4 (C-5), 96.5 (C-6), 164.2 (C-7), 94.8 (C8), 150.1 (C-9), 104.1 (C-10), 120.8 (C-1'), 130.1 (C-2', 6'), 115.1 $\left(\mathrm{C}-3^{\prime}, 5^{\prime}\right), 159.3\left(\mathrm{C}-4^{\prime}\right), 101.8\left(\mathrm{C}-1^{\prime \prime}\right), 69.7$ (C-2"), 70.1 (C-3"), 70.3 (C-4"), 71.1 (C-5"), 17.5 (C-6"), 55.8 (-OCH3).

Kaempferol-3-O- $\beta$-D-glucoside "astragalin" (8): yellow powder, ${ }^{1} \mathrm{H}$ NMR (DMSO- $\left.d 6,600 \mathrm{MHz}\right): \delta 6.44(1 \mathrm{H}, \mathrm{d}, J=1.98$ Hz, H-8), 6.25 (1H, d, $J=2.04$ Hz, H-6), 7.69 (2H, dd, $J=8.82$, Hz, H-2', 6'), 6.88 (1H, dd, $\left.J=8.88,2.64 \mathrm{~Hz}, \mathrm{H}-3^{\prime}, 5^{\prime}\right), 5.34(1 \mathrm{H}$, d, $\left.J=5.10 \mathrm{~Hz}, \mathrm{H}-1^{\prime \prime}\right), 3.53$ (1H, m, H-2"), 3.80 (1H, m, H-3"), 3.66 (1H, m, H-4"), 3.74 (1H, m, H-5"), 3.56 (1H, dd, $J=11.46,5.40$ Hz, H-6"a), 3.20 (1H, dd, $J=11.64,2.10 \mathrm{~Hz}, \mathrm{H}-6 " \mathrm{~b}) .{ }^{13} \mathrm{C}$ NMR (DMSO- $d 6,150 \mathrm{MHz}): \delta 156.6$ (C-2), 131.3 (C-3), 177.9 (C-4), 162.8 (C-5), 99.1 (C-6), 164.6 (C-7), 94.1 (C-8), 158.3 (C-9), 104.3 (C-10), $121.1\left(\mathrm{C}-1^{\prime}\right), 130.8$ (C-2', 6'), 115.7 (C-3', 5'), 160.4 $\left(\mathrm{C}-4^{\prime}\right),\left(\mathrm{C}-5^{\prime}\right), 101.6\left(\mathrm{C}-1^{\prime \prime}\right), 71.9\left(\mathrm{C}-2^{\prime \prime}\right), 56.2\left(\mathrm{C}-3^{\prime \prime}\right), 66.4\left(\mathrm{C}-4^{\prime \prime}\right)$, $71.2\left(\mathrm{C}-5^{\prime \prime}\right), 64.6\left(\mathrm{C}-6^{\prime \prime}\right)$.

Catechin (9): white needles, ${ }^{1} \mathrm{H}$ NMR (DMSO-d6, 600 MHz): $\delta 4.48$ (1H, d, $J=7.44 \mathrm{~Hz}, \mathrm{H}-2), 3.81(1 \mathrm{H}, \mathrm{m}, \mathrm{H}-3), 2.65$ (1H, dd, $J=16.12,5.45 \mathrm{~Hz}, \mathrm{H}-4 \mathrm{a}), 2.35$ (1H, dd, $J=16.14,8.35$ Hz, H-4b), 5.69 (1H, d, $J=2.1 \mathrm{~Hz}, \mathrm{H}-6), 5.89(1 \mathrm{H}, \mathrm{d}, J=2.1 \mathrm{~Hz}$, $\mathrm{H}-8), 6.72\left(1 \mathrm{H}, \mathrm{d}, J=1.88 \mathrm{~Hz}, \mathrm{H}-2^{\prime}\right), 6.68(1 \mathrm{H}, \mathrm{d}, J=8.34 \mathrm{~Hz}$, H-5') 6.59 ( $\left.1 \mathrm{H}, \mathrm{dd}, J=1.68,8.15 \mathrm{~Hz}, \mathrm{H}-6^{\prime}\right) .{ }^{13} \mathrm{C}$ NMR (DMSO- $d$, $150 \mathrm{MHz}): \delta 82.1$ (C-2), 66.3 (C-3), 27.9 (C-4), 156.2 (C-5), 95.2 (C-6), 156.5 (C-7), 93.2 (C-8), 155.4 (C-9), 99.1 (C-10), 130.6 (C-1'), 115.1 (C-2'), 144.9 (C-3'), 144.9 (C-4'), 114.5 (C-5'), $118.4\left(\mathrm{C}-6^{\prime}\right)$.

Epicatechin (10): white needles, ${ }^{1} \mathrm{H}$ NMR (DMSO- $d 6$, $600 \mathrm{MHz}): \delta 4.65$ (1H, d, J=4.64 Hz, H-2), 4.0 (1H, m, H-3), 2.65 $(1 \mathrm{H}, \mathrm{dd}, J=16.61,4.85 \mathrm{~Hz}, \mathrm{H}-4 \mathrm{a}), 2.45$ (1H, dd, $J=16.14,3.42$ Hz, H-4b), 5.72 (1H, d, $J=2.1 \mathrm{~Hz}, \mathrm{H}-6), 5.89$ (1H, d, $J=2.1 \mathrm{~Hz}$, H-8), 6.9 (1H, d, $\left.J=1.88 \mathrm{~Hz}, \mathrm{H}-2^{\prime}\right), 6.65$ (1H, d, $\left.J=8.34 \mathrm{~Hz}, \mathrm{H}-5^{\prime}\right)$, $6.59\left(1 \mathrm{H}, \mathrm{dd}, J=1.68,8.15 \mathrm{~Hz}, \mathrm{H}-6^{\prime}\right) .{ }^{13} \mathrm{C}$ NMR (DMSO- $d_{6}, 150$ $\mathrm{MHz}): \delta 78.1$ (C-2), 65.0 (C-3), 28.2 (C-4), 156.2 (C-5), 95.1 (C6), 156.5 (C-7), 94.1 (C-8), 155.8 (C-9), 98.2 (C-10), 130.6 (C-1'), 115.0 (C-2'), $144.4\left(\mathrm{C}-3^{\prime}\right), 144.5\left(\mathrm{C}-4^{\prime}\right), 114.7\left(\mathrm{C}-5^{\prime}\right), 118.0\left(\mathrm{C}-6^{\prime}\right)$.

Catechin-3-O-gallate (11): white amorphous powder, ${ }^{1} \mathrm{H}$ NMR (DMSO-d6, $600 \mathrm{MHz}): \delta 5.02(1 \mathrm{H}$, br.s, H-2), $5.34(1 \mathrm{H}, \mathrm{m}$, H-3), 2.67 (1H, d, $J=16.9 \mathrm{~Hz}, \mathrm{H}-4 \mathrm{a}), 2.93$ (1H, dd, $J=16.9,4.50$ $\mathrm{Hz}, \mathrm{H}-4 \mathrm{~b}), 5.93$ (1H, d, $J=1.96 \mathrm{~Hz}, \mathrm{H}-6), 5.83$ (1H, d, $J=1.96$ $\mathrm{Hz}, \mathrm{H}-8), 6.85\left(1 \mathrm{H}, \mathrm{d}, J=2.2 \mathrm{~Hz}, \mathrm{H}-2^{\prime}\right), 6.65(1 \mathrm{H}, \mathrm{d}, J=8.4 \mathrm{~Hz}$, H-5') 6.75 (1H, dd, $\left.J=8.4,2.20 \mathrm{~Hz}, \mathrm{H}-6^{\prime}\right), 6.82$ (2H, s, H-2", 6"). ${ }^{13} \mathrm{C}$ NMR (DMSO- $\left.d, 150 \mathrm{MHz}\right): \delta 76.5(\mathrm{C}-2), 68.2(\mathrm{C}-3), 25.7$ (C-4), 156.5 (C-5), 95.5 (C-6), 156.6 (C-7), 94.4 (C-8), 155.6 (C9), 97.3 (C-10), $129.4\left(\mathrm{C}-1^{\prime}\right), 114.3$ (C-2'), 144.7 (C-3'), 144.7 (C$\left.4^{\prime}\right), 115.1\left(\mathrm{C}-5^{\prime}\right), 117.6\left(\mathrm{C}-6^{\prime}\right), 119.2\left(\mathrm{C}-1^{\prime \prime}\right), 108.6\left(\mathrm{C}-2^{\prime \prime}\right), 145.4$ (C-3"), $138.6\left(\mathrm{C}-4^{\prime \prime}\right), 145.4\left(\mathrm{C}-5^{\prime \prime}\right), 108.6\left(\mathrm{C}-6^{\prime \prime}\right), 165.2(\mathrm{C}=\mathrm{O})$.

5-O-trans-p-coumaroylquinic acid methyl ester (12): pale yellow powder, ${ }^{1} \mathrm{H}$ NMR (DMSO- $\left.d, 600 \mathrm{MHz}\right): \delta 1.93(1 \mathrm{H}$, dd, $J=14.21,3.42 \mathrm{~Hz}, \mathrm{H}-2 \mathrm{a}), 2.08(1 \mathrm{H}, \mathrm{dd}, J=14.21,4.55 \mathrm{~Hz}$, H-2b), 3.87 (1H, m, H-3), 3.56 (1H, m, H-4), 5.01 (1H, m, H-5), 1.75 (1H, m, H-6a), 2.06 (1H, m, H-6b), $7.52(2 \mathrm{H}, \mathrm{d}, J=8.44 \mathrm{~Hz}$, H-2', 6'), 6.79 (2H, d, $\left.J=8.44 \mathrm{~Hz}, \mathrm{H}-3^{\prime}, 5^{\prime}\right), 7.44(1 \mathrm{H}, \mathrm{d}, J=16.44$ Hz, H-7'), 6.24 (1H, d, J=16.22 Hz, H-8'), 3.54 (3H, s, -OCH3). ${ }^{13} \mathrm{C}$ NMR (DMSO- $\left.d_{6}, 150 \mathrm{MHz}\right): \delta 73.0(\mathrm{C}-1), 34.8$ (C-2), 66.9 (C-3), 69.1 (C-4), 70.7 (C-5), 36.9 (C-6), 173.9 (C-7), 124.8 
$\left(\mathrm{C}-1^{\prime}\right), 129.9\left(\mathrm{C}-2^{\prime}\right), 115.6\left(\mathrm{C}-3^{\prime}\right), 159.8\left(\mathrm{C}-4^{\prime}\right), 115.6\left(\mathrm{C}-5^{\prime}\right), 129.9$ (C-6'), 144.7 (C-7'), $113.8\left(\mathrm{C}-8^{\prime}\right), 165.4\left(\mathrm{C}-9^{\prime}\right), 51.4(-\mathrm{OCH} 3)$.

5-O-caffeoylquinic acid methyl ester (13): pale yellow powder, ${ }^{1} \mathrm{H}$ NMR (DMSO- $d, 600 \mathrm{MHz}$ ): $\delta 1.93(1 \mathrm{H}, \mathrm{dd}, J=$ 14.11, 3.42 Hz, H-2a), 2.10 (14.21, 4.45 Hz, H-2b), 3.81 (1H, m, H-3), 3.56 (1H, m, H-4), 5.00 (1H, m, H-5), 1.76 (1H, m, H-6a), $2.10(1 \mathrm{H}, \mathrm{m}, \mathrm{H}-6 \mathrm{~b}), 7.03\left(1 \mathrm{H}, \mathrm{d}, J=2.1 \mathrm{~Hz}, \mathrm{H}-2^{\prime}\right), 6.77(1 \mathrm{H}, \mathrm{d}$, $\left.J=8.34 \mathrm{~Hz}, \mathrm{H}-5^{\prime}\right), 6.97$ (1H, dd, $\left.J=8.34,1.96 \mathrm{~Hz}, \mathrm{H}-6^{\prime}\right), 7.38$ $\left(1 \mathrm{H}, \mathrm{d}, J=16.1 \mathrm{~Hz}, \mathrm{H}-7^{\prime}\right), 6.10\left(1 \mathrm{H}, \mathrm{d}, J=16.1 \mathrm{~Hz}, \mathrm{H}-8^{\prime}\right), 3.56$ $(3 \mathrm{H}, \mathrm{s},-\mathrm{OCH} 3) .{ }^{13} \mathrm{C}$ NMR (DMSO- $\left.d, 150 \mathrm{MHz}\right): \delta 73.3(\mathrm{C}-1)$, 35.0 (C-2), 66.7 (C-3), 69.3 (C-4), 71.0 (C-5), 37.4 (C-6), 173.6 (C-7), 125.4 (C-1'), 114.6 (C-2'), 145.7 (C-3'), 148.5 (C-4'), 115.9 (C-5'), $121.4\left(\mathrm{C}-6^{\prime}\right), 145.2\left(\mathrm{C}-7^{\prime}\right), 113.9\left(\mathrm{C}-8^{\prime}\right), 165.4\left(\mathrm{C}-9^{\prime}\right), 51.8$ (-OCH3).

\section{Molecular docking and simulations of binding activity}

Molecular operating environment (MOE) program 2008.10 was used for the optimization of both examined ligands and receptors for docking studies. Molecular docking was carried out to investigate the affinity of isolated compounds to COX-2 (PDB ID: 3NL1) complexed with celecoxib and VEGFR-2 (PDB ID: 4ASD) complexed with sorafenib retrieved from the Protein Data Bank (http://www.rcsb.org/pdb) at the molecular level (Abdellatif et al., 2020; Bernstein et al., 1977). The downloaded proteins were prepared for docking by 3D protonation, deleting of unnecessary water molecules and all co-crystallized ligands and metals, and receptor fixation. Furthermore, the isolated compounds were subjected to 3D generation and energy minimization using Merck Molecular Forcefield (MMFF94s) to a gradient 0.05 . The adopted docking procedure followed the standard protocol implemented in MOE 2008.10 and the geometry of the resulting complexes was studied using MOE's Pose Viewer utility. The interaction between the ligands and receptors binding site was generated and the results of docking were recorded as pose score $(S)$ and binding energy.

\section{RESULTS AND DISCUSSION}

\section{Identification of isolated compounds}

Phytochemical investigation of the methanolic extract of the flowers led to the isolation and characterization of 13 compounds isolated for the first time from this plant species. One-dimensional and two-dimensional NMR together were used for structural elucidation of the isolated compounds and in comparison, with previously reported spectral data. Accordingly, the isolated compounds were identified as quercetin (1) (Jaiswal et al., 2012, Jaiswal et al., 2014), quercitrin (2) (Dai and Yu, 2005, Jaiswal et al., 2012), avicularin (3) (Manivannan and Shopna, 2015), taxifolin-3-O- $\beta$-L-arabinoside (4) (Jin et al., 2009), azalein (5) (Hang et al., 2011), kaempferol-3-O- $\alpha$-L-rhamnoside (6), kaempferol-4'-methoxy-3-O- $\alpha$-L-rhamnoside (7) (Jaiswal et al., 2014), kaempferol-3-O- $\alpha$-D-glucoside (8) (Hong et al., 2007, Jaiswal et al., 2014), catechin (9) (Jaiswal et al., 2012; Jin et al., 2009), epicatechin (10) (Jaiswal et al., 2012), catechin gallate (11) (Jaiswal et al., 2012; Kemertelidze et al., 2007), 5-O-p-transcoumaroylquinic acid methyl ester (12) (Jaiswal et al., 2011), and 5-O-caffeoylquinic acid methyl ester (13) (Jaiswal et al., 2011). The data of the isolated compounds are listed in detail in the experimental section. The chemical structures of isolated compounds are shown in Figure 1.

\section{Molecular docking of isolated compounds}

Recently, molecular docking was considered an important tool for the discovery of new biologically active and lead compounds (ligand) that have specific affinity to targeted proteins (enzyme or receptor) of known three-dimensional structure (Meng et al., 2011). The docking simulation technique aims to predict the<smiles>[R10]Oc1ccc(-c2oc3cc(O)cc(O[R4])c3c(=O)c2O[R2])cc1[Y3]</smiles>

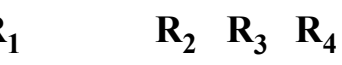

(1) $\mathrm{H}$

(2) Rhamnoside

(3) Arabinofuranoside

(4) Arabinopyranoside

(5)

(6)

(7)

(8)

$$
\mathrm{H} \quad \mathrm{OH} \quad \mathrm{H}
$$

$\begin{array}{lll}\mathrm{H} & \mathrm{OH} & \mathrm{H}\end{array}$

$\mathrm{H} \quad \mathrm{OH} \quad \mathrm{H}$

$\begin{array}{lll}\mathrm{H} & \mathrm{OH} & \mathrm{H}\end{array}$

Rhamnoside

$\mathrm{CH}_{3} \mathrm{OH}$

Rhamnoside

$\mathrm{H} \quad \mathrm{H}$

Rhamnoside

$\begin{array}{lll}\mathrm{H} & \mathrm{H} & \mathrm{CH}_{3}\end{array}$ Glucoside

$\begin{array}{lll}\mathrm{H} & \mathrm{H}\end{array}$<smiles>Oc1cc(O)c2c(c1)O[C@H](c1ccc(O)c(O)c1)[C@H](O)C2</smiles>

(9)<smiles>Oc1cc(O)c2c(c1)O[C@H](c1ccc(O)c(O)c1)[C@H](O)C2</smiles>

(10)<smiles>[R][R]=O</smiles>

Figure 1. The structures of isolated compounds (1-13). 
Table 1. Docking poses' scores of isolated compounds from $R$. yunnanense against COX-2 (3NL1) receptor.

\begin{tabular}{|c|c|c|c|c|c|}
\hline No. & Ligand no. & $\mathrm{S}(\mathrm{kcal} / \mathrm{mol})$ & E_conf. & No. & $\begin{array}{l}\text { Hydrogen bond } \\
\text { Interacting residues (no. of bonds) }\end{array}$ \\
\hline 1 & 1 & -15.5926 & 0.00 & 8 & Gln178 (1), His75 (2), Ser516 (2), and Tyr341 (3) \\
\hline 2 & 2 & -19.4542 & 0.60 & 12 & $\begin{array}{c}\operatorname{Arg} 106 \text { (3), Arg499 (3), Gln178 (1), Glu510 (1), Leu338 (1), Pro71 (1), } \\
\text { and Tyr341 (2) }\end{array}$ \\
\hline 3 & 3 & -19.0703 & 0.00 & 8 & $\operatorname{Arg} 106(1), \operatorname{His} 75$ (2), Ser516 (2), and Tyr341 (3) \\
\hline 4 & 4 & -20.4075 & 0.46 & 12 & $\operatorname{Arg} 106$ (2), Gln178 (1), His75 (2), Ser516 (2), and Tyr341 (5) \\
\hline 5 & 5 & -18.8015 & 0.80 & 5 & $\operatorname{Arg} 106(2), \operatorname{Ser} 516(1)$, and $\operatorname{Tyr} 341$ (2) \\
\hline 6 & 6 & -18.1847 & 0.00 & 2 & Arg499 and Leu338 \\
\hline 7 & 7 & -17.8494 & 1.00 & 5 & $\operatorname{Arg} 106(1), \operatorname{Arg} 499(1), \operatorname{Gln} 178(1)$, and Tyr341 (2) \\
\hline 8 & 8 & -18.5687 & 0.60 & 6 & $\operatorname{Arg} 106$, Gln178, Tyr341 (2), and Tyr371 (2) \\
\hline 9 & 9 & -14.7821 & 0.00 & 7 & Gln178 (1), His75 (2), Ser516 (2), and Tyr341 (2) \\
\hline 10 & 10 & -15.1491 & 0.40 & 6 & $\operatorname{Arg} 499$ (1), Leu338 (1), Ser516 (2), and Tyr341 (2) \\
\hline 11 & 11 & -19.6792 & 0.00 & 4 & $\operatorname{Arg} 499(1), \operatorname{Gln} 178(1)$, and Ser516 (2) \\
\hline 12 & 12 & -15.1796 & 0.00 & 4 & Gln178 (1), Ser516 (2), and Tyr341 (1) \\
\hline 13 & 13 & -15.3721 & 1.00 & 5 & Arg106 (1), Arg499 (1), Phe504 (2), and Tyr341 (1) \\
\hline 14 & Celecoxib & -13.1283 & 0.00 & 4 & Arg106 (1), Glu510 (1), His75 (1), and Tyr341 (1) \\
\hline
\end{tabular}

Table 2. Docking poses' scores of isolated compounds from $R$. yunnanense against VEGFR-2 (4ASD) receptor.

\begin{tabular}{cccccc}
\hline No. & Ligand no. & S (kcal/mol) & E_conf. & No. & $\begin{array}{c}\text { Hydrogen bond } \\
\text { Interacting residues (no. of bonds) }\end{array}$ \\
\hline 1 & 1 & -16.7320 & 0.00 & 6 & Asp1046 (1), Cys919 (1), Glu885 (1), Glu917 (1), and Lys868 (2) \\
2 & 2 & -17.6036 & 0.87 & 7 & Asp1046 (2), Asp814 (1), Glu885 (1), HOH2142 (2), and Ile1044 (1) \\
3 & 3 & -16.3620 & 0.08 & 11 & Asp1046 (3), Asp814 (1), Glu885 (1), HOH2142 (4), and Lys868 (2) \\
4 & 4 & -14.6453 & 0.00 & 8 & Arg1027 (2), His1026 (2), Ile1025 (3), and Leu1049 (1) \\
5 & 5 & -14.2163 & 0.20 & 5 & Asp1046 (2), HOH2142 (1), and Lys868 (2) \\
6 & 6 & -15.8128 & 0.60 & 7 & Asp1046 (2), Glu885 (1), HOH2142 (2), Lys868 (1), and Val899 (1) \\
7 & 7 & -14.3816 & 0.80 & 4 & Glu885, Lys868, Asp1046, and HOH2142 \\
9 & 8 & -15.4336 & 0.60 & 5 & Glu885, HOH2142 (2), and Lys868 (2) \\
10 & 9 & -16.5239 & 0.00 & 7 & Asp1046 (1), Cys919 (2), Glu917 (1), HOH2142 (2), and Lys868 (1) \\
11 & 10 & -15.9363 & 0.40 & 6 & Asp1046 (1), Cys919 (2), Glu917 (1), and HOH2142 (2) \\
12 & 11 & -15.0099 & 0.00 & 7 & Arg1027 (1), Asp1046 (1), Ile1025 (1), Ile1044 (1), Lys868 (1), and \\
13 & 12 & -13.3659 & 0.00 & 5 & Val899 (2) \\
14 & 13 & -16.3042 & 1.40 & 7 & Asp1046 (1), Cys919 (2), Glu885 (1), HOH2142 (2), and Lys868 (1) \\
\hline
\end{tabular}

pose, experimental binding modes, and binding affinity of ligand within the targeted receptor-binding site (Guedes et al., 2014).

Herein, we examined the isolated compounds (1-13) by the molecular docking technique to identify their potential as antiangiogenetic and anti-inflammatory. We utilized COX-2 (PDB ID: 3NL1) as representative proteins for inflammation and VEGFR-2 (PDB ID: 4ASD) for angiogenesis. The pose scoring, hydrogen bonding and interacting residues for these compounds with selected proteins were listed in Tables 1 and 2 .

The analysis of docking results for the isolated compound with COX-2 receptor (Table 1, Fig. 2) revealed that all the tested compounds exhibited binding affinity to the receptor ranges from $-20.4075 \mathrm{kcal} / \mathrm{mol}$ for compound 4 to $-14.7821 \mathrm{kcal} /$ mol for compound 9, which was considered more active than the 
standard anti-inflammatory COX-2 inhibitor compound celecoxib $(-13.1283 \mathrm{kcal} / \mathrm{mol})$.

Furthermore, the results of interactions with VEGFR-2 showed that compound 2 had the most stable binding energy to allosteric site of VEGFR-2 with a score of $-17.6036 \mathrm{kcal} / \mathrm{mol}$ in comparison to sorafenib $(-16.6507 \mathrm{kcal} / \mathrm{mol})$ as the standard inhibitor to angiogenesis (Table 2, Fig. 2).

Compound 4 showed the best binding affinity to COX-2 receptor by forming 12 hydrogen bonds with Arg106 (2), Gln178 (1), His75 (2), Ser516 (2), and Tyr341 (5) amino acid residues with bond length ranging from 2.14 to $3.11 \AA$, in addition to other hydrophobic interactions essential for affinity (Fig. 3).

Furthermore, compound 2 revealed the highest binding affinity to VEGR-2 receptor site by interaction through hydrogen bonding and hydrophobic interactions with the amino acid residues from the receptor pocket (Fig. 4).

It noteworthy that the quercitrin (2) showed predominant activity for the two examined receptors [COX-2

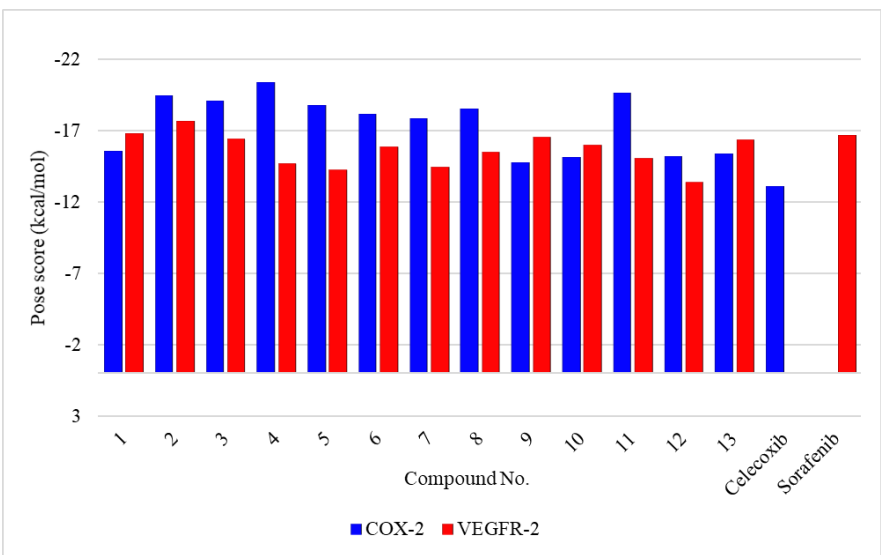

Figure 2. Pose score of isolated compounds' interactions with COX-2 and VEGFR-2 receptor in comparison with celecoxib and sorafenib.

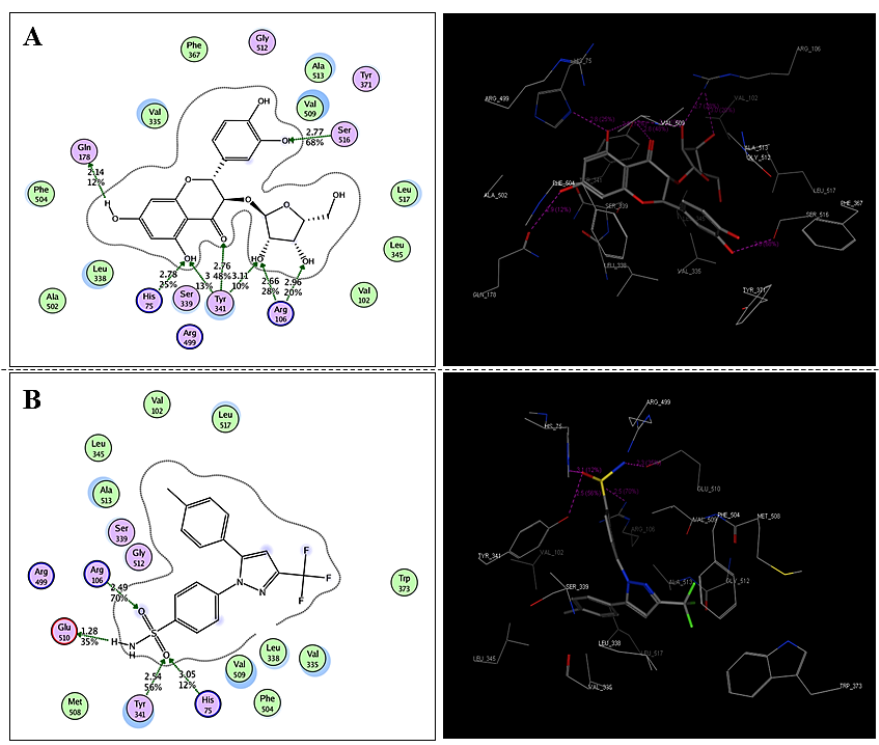

Figure 3. 2D and 3D ligand interactions of compound 4 (A) and celecoxib (B) with COX-2 receptor.
$(-19.4542 \mathrm{kcal} / \mathrm{mol})$ and VEGFR-2 (-17.6036 kcal $/ \mathrm{mol})]$ (Fig. 5) more than the compared standards [celecoxib $(-13.1283$ $\mathrm{kcal} / \mathrm{mol})$ and sorafenib $(-16.6507 \mathrm{kcal} / \mathrm{mol})$ ] which could be a lead compound for the development of dual acting compounds for the treatment of tumors either directly by antiangiogenic activity or by acting on inflammatory mediators as predisposing factors for tumor prognosis and development. The results are in agreement with previously reported activities of quercitrin (2) as an antioxidant, anti-inflammatory, anti-proliferative, antiangiogenic, and apoptotic inducer. These effects are directly linked to the anti-tumor property by its interference with receptors, cellular enzymes, and signal transduction systems (Ezzati et al., 2020; Stochmalová et al., 2013). The comparison between the interactions of compound 2 with the two receptors (Fig. 5) revealed that the hydroxyl group at 4' position of ring $\mathrm{B}$ and the oxygen group in 3 position of flavonol together with oxygen groups of positions 1, 2, and 3 of the rhamnose moiety are essential for the interaction by hydrogen bonding for both receptors.
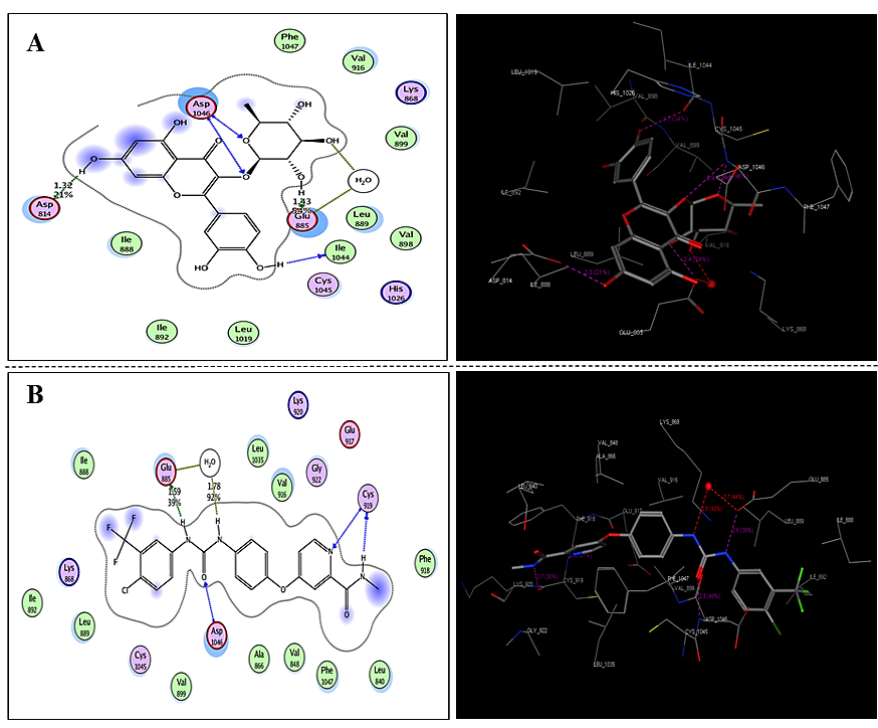

Figure 4. 2D and 3D ligand interactions of compound 2 (A) and sorafenib (B) with VEGFR-2 receptor.

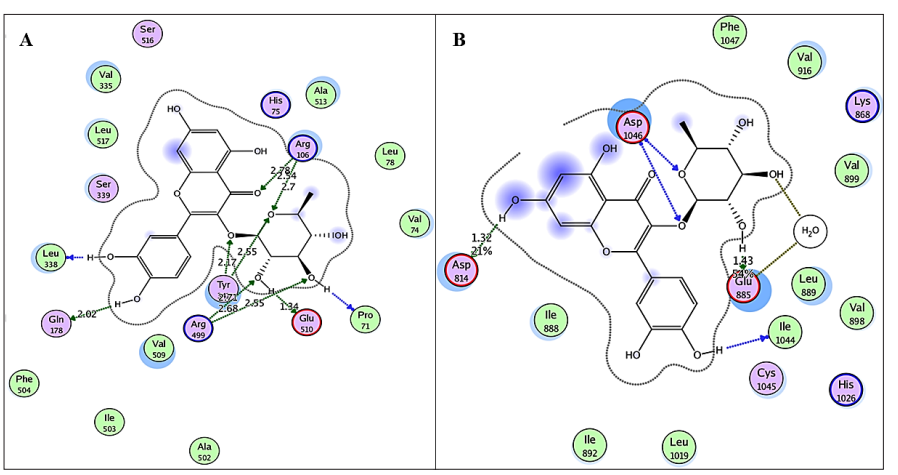

Figure 5. 2D ligand interactions of compound 2 (quercitrin) with COX-2 (A) and VEGFR-2 receptor (B). 


\section{CONCLUSION}

This work revealed that $R$. yunnanense Franch. is a rich source for phenolic and flavonoid constituents. Thirteen phenolic compounds were isolated and identified from the methanolic extract of the plant flowers for the first time. Molecular docking studies of the compounds 1-13 into the active sites of COX2 and VEGFR-2 receptors revealed good fitting into the active site of both enzymes. Quercitrin (2) exhibited significant activity against the tested receptors in comparison with the relevant standards. Regarding these results, quercitrin (2) could be used to design potent antitumor agents that could be directly act by antiangiogenic activity or by acting on inflammatory mediators as predisposing factor for tumor prognosis and development.

\section{ABBREVIATIONS}

VEGFR-2 Vascular endothelial growth factor receptor-2 COX-2 Cyclooxygenase-2

NMR Nuclear magnetic resonance

HPLC High-pressure liquid chromatography

$\mathrm{MeOH} \quad$ Methanol

\section{ACKNOWLEDGMENT}

The authors thank Prof. Marcel Jaspars, Marine Biodiscovery Centre, University of Aberdeen, UK, for the NMR analysis of our samples.

\section{FUNDING}

There is no funding to report.

\section{CONFLICTS OF INTEREST}

The authors report no financial or any other conflicts of interest in this work.

\section{ETHICAL APPROVALS}

This study does not involve experiments on animals or human subjects.

\section{PUBLISHER'S NOTE}

This journal remains neutral with regard to jurisdictional claims in published institutional affiliation.

\section{AUTHOR'S CONTRIBUTIONS}

All authors contributed to concept and design, interpretation, data analysis, funding, and final approval. Reda Abdelhamid, Mohamed Abouelela, and Marwa Hassan contributed to writing the original draft and statistical analysis. Mohamed Abdelkader and Mostafa Rateb contributed to conceptualization, data acquisition, revision of the manuscript, technical material support, and supervision.

\section{REFERENCES}

Abdellatif KR, Abdelall EK, Lamie PF, Labib MB, El-Nahaas E-S, Abdelhakeem MM. New pyrazole derivatives possessing amino/ methanesulphonyl pharmacophore with good gastric safety profile: design, synthesis, cyclooxygenase inhibition, anti-inflammatory activity and histopathological studies. Bioorg Chem, 2020; 95:103540-53.

Abhinand CS, Athira PA, Soumya SJ, Sudhakaran PR. Multiple targets directed multiple ligands: an in silico and in vitro approach to evaluating the effect of triphala on angiogenesis. Biomolecules, 2020; 10(2):177-93.
Adhikari DP, Schutzki RE, DeWitt DL, Nair MG. Effects of amelanchier fruit isolates on cyclooxygenase enzymes and lipid peroxidation. Food Chem, 2006; 97(1):56-64.

Ahmad VU, Ullah F, Hussain J, Farooq U, Zubair M, Khan MTH, Choudhary MI. Tyrosinase inhibitors from Rhododendron collettianum and their structure-activity relationship (SAR) studies. Chem Pharm Bull, 2004 52(12):1458-61.

Al Abd AM, Alamoudi AJ, Abdel-Naim AB, Neamatallah TA, Ashour OM. Anti-angiogenic agents for the treatment of solid tumors: potential pathways, therapy and current strategies-a review. J Adv Res, 2017; 8(6):591-605.

Asuzu PC, Besong SA, Aryee AN. Polyphenols and other phytochemicals in cancer prevention and management. The FASEB J, 2019; 33(S1):80243-3.

Bahamonde SMA, Flores ML, Córdoba OL, Taira CA, Gorzalczany S. Antinociceptive and anti-inflammatory activities of an aqueous extract of Chiliotrichum diffusum. Rev Bras Farmacogn, 2013; 23(4):699-705.

Bernstein FC, Koetzle TF, Williams GJ, Meyer Jr EF, Brice MD, Rodgers JR, Kennard O, Shimanouchi T, Tasumi M. The Protein Data Bank: a computer-based archival file for macromolecular structures. Europ J Biochem, 1977; 80(2):319-24.

Chhetri HP, Yogol NS, Sherchan J, Anupa K, Mansoor S, Thapa P. Phytochemical and antimicrobial evaluations of some medicinal plants of Nepal. KUSET, 2008; 4(1):49-54.

Dai S, Yu D. Studies on the flavonoids in stem of Rhododendron anthopogonoide II. China j Chinese Materia Medica, 2005; 30(23):1830-3.

Ezzati M, Yousefi B, Velaei K, Safa A. A review on anticancer properties of quercetin in breast cancer. Life Sci, 2020; 248:117463-75.

Fosslien E. Biochemistry of cyclooxygenase (COX)-2 inhibitors and molecular pathology of COX-2 in neoplasia. Crit Rev Clin Lab Sci, 2000; 37(5):431-502.

Guedes IA, de Magalhães CS, Dardenne LE. Receptor-ligand molecular docking. Biophys Rev, 2014; 6(1):75-87.

Hang NTT, Miyajima I, Ureshino K, Kobayashi N, Kurashige Y, Matsui T, Okubo H. Anthocyanins of wild Rhododendron simsii Planch. flowers in Vietnam and Japan. J Jpn Soc Hortic Sci, 2011; 80(2):206-13.

Hong HS, Jeon SH, Kwon YS. Cytotoxic constituents from the stem of Rhododendron mucronulatum. Korean J Pharmacogn, 2007; 38(3):227-33

Jafari S, Saeidnia S, Abdollahi M. Role of natural phenolic compounds in cancer chemoprevention via regulation of the cell cycle. Curr Pharm Biotechnol, 2014; 15(4):409-21.

Jaiswal R, Deshpande S, Kuhnert N. Profiling the chlorogenic acids of Rudbeckia hirta, Helianthus tuberosus, Carlina acaulis and Symphyotrichum novae-angliae leaves by LC-MSn. Phytochem Anal, 2011; 22(5):432-41.

Jaiswal R, Jayasinghe L, Kuhnert N. Identification and characterization of proanthocyanidins of 16 members of the Rhododendron genus (Ericaceae) by tandem LC-MS. J Mass Spectrom, 2012; 47(4):502-15.

Jaiswal R, Karar MGE, Gadir HA, Kuhnert N. Identification and characterisation of phenolics from Ixora coccinea L.(Rubiaceae) by liquid chromatography multi-stage mass spectrometry. Phytochem Anal, 2014; 25(6):567-76.

Jin C, Strembiski W, Kulchytska Y, Daneshtalab RGMM. Flavonoid glycosides from Ledumpalustre L. Subsp. decumbens (Ait) Hulton. DARU J Pharm Sci, 1999; 7(4):5-8.

Jin HZ, Chen G, Li XF, Shen YH, Yan SK, Zhang L, Yang M, Zhang WD. Flavonoids from Rhododendron decorum. Chem Nat Compd, 2009; 45(1):85-6.

Kemertelidze E, Shalashvili K, Korsantiya B, Nizharadze N, Chipashvili NS. Therapeutic effect of phenolic compounds isolated from Rhododendron ungernii leaves. Pharm Chem J, 2007; 41(1):10-3. 
Ko J, Auyeung K. Target-oriented mechanisms of novel herbal therapeutics in the chemotherapy of gastrointestinal cancer and inflammation. Curr Pharm Des, 2013; 19(1):48-66.

Leahy KM, Ornberg RL, Wang Y, Zweifel BS, Koki AT, Masferrer JL. Cyclooxygenase-2 inhibition by celecoxib reduces proliferation and induces apoptosis in angiogenic endothelial cells in vivo. Cancer Res, 2002; 62(3):625-31.

Liu XH, Kirschenbaum A, Yao S, Lee R, Holland JF, Levine AC. Inhibition of cyclooxygenase-2 suppresses angiogenesis and the growth of prostate cancer in vivo. J Urol, 2000; 164(3 Part 1):820-5.

Löhr G, Beikler T, Hensel A. Inhibition of in vitro adhesion and virulence of Porphyromonas gingivalis by aqueous extract and polysaccharides from Rhododendron ferrugineum L. A new way for prophylaxis of periodontitis? Fitoterapia, 2015; 107:105-13.

Manivannan R, Shopna R. Isolation of quercetin and isorhamnetin derivatives and evaluation of anti-microbial and anti-inflammatory activities of Persicaria glabra. Nat Prod Sci, 2015; 21(3):170-5.

Meng XY, Zhang HX, Mezei M, Cui M. Molecular docking: a powerful approach for structure-based drug discovery. Curr Comput Aided Drug Des, 2011; 7(2):146-57.

Noreen Y, Serrano G, Perera P, Bohlin L. Flavan-3-ols isolated from some medicinal plants inhibiting COX-1 and COX-2 catalysed prostaglandin biosynthesis. Planta Med, 1998; 64(06):520-4.

Orhan I, Şener B, Choudhary M, Khalid A. Acetylcholinesterase and butyrylcholinesterase inhibitory activity of some Turkish medicinal plants. J Ethnopharmacol, 2004; 91(1):57-60.

Popescu R, Kopp B. The genus Rhododendron: an ethnopharmacological and toxicological review. J Ethnopharmacol, 2013; 147(1):42-62.

Rateb ME, Hassan HM, Arafa El SA, Jaspars M, Ebel R. Decorosides A and B, cytotoxic flavonoid glycosides from the leaves of Rhododendron decorum. Nat Prod Commun, 2014; 9(4):473-6.

Riaz A, Rasul A, Hussain G, Zahoor MK, Jabeen F, Subhani Z, Younis T, Ali M, Sarfraz I, Selamoglu Z. Astragalin: a bioactive phytochemical with potential therapeutic activities. Adv Pharmacol Sci, 2018; 2018:1-5.

Roleira FM, Tavares-da-Silva EJ, Varela CL, Costa SC, Silva T, Garrido J, Borges F. Plant derived and dietary phenolic antioxidants: anticancer properties. Food Chem, 2015; 183:235-58.

Sayed AM, Khattab AR, AboulMagd AM, Hassan HM, Rateb ME, Zaid H, Abdelmohsen UR. Nature as a treasure trove of potential antiSARS-CoV drug leads: a structural/mechanistic rationale. RSC Adv, 2020; 10(34):19790-802.

Silici S, Sagdic O, Ekici L. Total phenolic content, antiradical, antioxidant and antimicrobial activities of Rhododendron honeys. Food Chem, 2010; 121(1):238-43.

Stochmalová A, Sirotkin A, Kádasi A, Alexa R. Physiological and medical effects of plant flavonoid quercetin. J Microbiol Biotechnol Food Sci, 2013; 2(1):1915-26.

Sung MS, Lee EG, Jeon HS, Chae HJ, Park SJ, Lee YC, Yoo WH. Quercetin inhibits IL-1 $\beta$-induced proliferation and production of MMPs, COX-2, and PGE2 by rheumatoid synovial fibroblast. Inflammation, 2012; 35(4):1585-94
Takahashi H, Hirata S, Minami H, Fukuyama Y. Triterpene and flavanone glycoside from Rhododendron simsii. Phytochemistry, 2001; 56(8):875-9.

Tasdemir D, Brun R, Perozzo R, Dönmez A. Evaluation of antiprotozoal and plasmodial enoyl-ACP reductase inhibition potential of turkish medicinal plants. Phytother Res, 2005; 19(2):162-6.

Toomey D, Murphy J, Conlon K. COX-2, VEGF and tumour angiogenesis. The Surgeon, 2009; 7(3):174-80.

Ullah F, Hussain H, Hussain J, Bukhari IA, Khan MTH, Choudhary MI, Gilani AH, Ahmad VU. Tyrosinase inhibitory pentacyclic triterpenes and analgesic and spasmolytic activities of methanol extracts of Rhododendron collettianum. Phytother Res, 2007; 21(11):1076-81.

Valero MS, González M, Ramón-Gimenez M, Andrade PB, Moreo E, Les F, Fernandes F, Gómez-Rincón C, Berzosa C, de Jalón JAG. Jasonia glutinosa (L.) DC., a traditional herbal medicine, reduces inflammation, oxidative stress and protects the intestinal barrier in a murine model of colitis. Inflammopharmacology, 2020; 28(6):1717-34.

Verma N, Singh AP, Amresh G, Sahu P, Rao CV. Antiinflammatory and anti-nociceptive activity of Rhododendron arboreum. J Pharm Res, 2010; 3(6):1376-80.

Wang Z, Li F, Quan Y, Shen J. Avicularin ameliorates human hepatocellular carcinoma via the regulation of NF-кB/COX-2/PPAR- $\gamma$ activities. Mol Med Rep, 2019; 19(6):5417-23.

Wong RS. Role of nonsteroidal anti-inflammatory drugs (NSAIDs) in cancer prevention and cancer promotion. Adv Pharmacol Sci, 2019; 2019:1-0.

Zhang Z, Lv G, Du L. Avicularin reduces the expression of mediators of inflammation and oxidative stress in bradykinin-treated MG-63 human osteoblastic osteosarcoma cells. Med Sci Monit, 2020; 26:e921957-1.

Zheng M. An experimental study of the anti-HSV-II action of 500 herbal drugs. J Tradit Chin Med, 1989; 9(2):113-6.

How to cite this article:

Abdelkader MSA, Abdelhamid RA, Abouelela ME, Rateb ME, Ahmed MH. Isolation of phenolic constituents from Rhododendron yunnanense flowers as a potent cyclooxygenase-2 and vascular endothelial growth factor receptor-2 inhibitor: Phytochemical and molecular simulation studies. Indonesia. J Appl Pharm Sci, 2021; 11(11):087-094. 